\title{
MODELAGEM DINÂMICA DE BACIA HIDROGRÁFICA: AVALIAÇÃO DOS COMPONENTES MANGANÊS E FÓSFORO TOTAL NO RIO DAS MORTES, MG
}

\author{
BERNARDINO, A. D.*; FORTES, M. \& FERREIRA, W. R. \\ Centro Universitário UNA \\ *Corresponding author: durco.aluizio@gmail.com
}

\begin{abstract}
Dynamic Modeling of of Watersheds: Evaluation of total Manganese and Phosphorus in Rio das Mortes, MG (2013) Braz. J. Aquat. Sci. Technol. 17(1):43-51. DOI:10.14210/bjast.v17.n1.p43-51. elSSN 1983-9057. The rate of water resource self-depuration is limited, mainly due to the associated pollutant load. To minimize inherent quality problems, many countries, including Brazil, have adopted Regulatory Decisions targeted at water resource quality. Thus, in this context, this study presents a dynamic model developed to analyze the interrelationships between the flow rate and the concentration of pollutants in the watershed of the Rio das Mortes, Minas Gerais State, Brazil. The methodology was based on the STELLA, a software that allows system dynamic analysis. Official data from the 2000-2009 period, related to the hydrological behavior of monthly average flow rates and pollutant concentrations of Total Manganese and Total Phosphorus allowed simulating and effectuating a sensitivity analysis of the possible effects of changes in pollutant concentrations, in that watershed. The model developed in this study allowed a better understanding of the behavior of the watershed with respect to temporal and spatial pollution changes.
\end{abstract}

Keywords: water pollution; environmental impact; dynamic analysis of water pollution; Total Manganese; Total Phosphorus.

INTRODUÇÃO

O ecossistema aquático é o mais afetado do planeta. Devido aos diversos usos do solo, é muito explorado pelas atividades humanas, como a irrigação, geração de eletricidade e eliminação de resíduos. Os cursos de água (rios, córregos, ribeirões, lagos e lagoas) possuem contato com estas atividades devido aos sistemas de captação e despejo de efluentes que alteram diretamente suas características (Malmqvist \& Rundle, 2002).

Conforme estes autores os diversos usos do solo vêm causando um aumento das concentrações de poluentes químicos inorgânicos e orgânicos nos rios. Esta poluição varia conforme o desenvolvimento das atividades humanas existentes no entorno do rio. Uma redução da concentração de metais e compostos orgânicos tóxicos vem sendo notada nos últimos 30 anos, mas há um aumento da presença de nutrientes nestes rios.

O aumento da contaminação dos recursos hídricos em todo o mundo por inúmeros compostos químicos industriais e naturais é um dos principais problemas ambientais que a humanidade enfrenta. Estes compostos, em muitos casos, estão presentes em baixas concentrações, mas alguns deles devem ser considerados importantes pela sua concentração e capacidade toxicológica (Schwarzenbach et al., 2006).

Os ecossistemas aquáticos recebem uma carga constante de poluentes que causam danos às áreas de inundação e consequentemente podem atingir as regiões costeiras, prejudicando outros ecossistemas. Em vários rios existe um sistema de monitoramento para apresentar as concentrações de poluentes, nutrientes e compostos tóxicos. Esta rede de monitoramento vem indicando que está ocorrendo um aumento destes compostos, principalmente de nutrientes como o nitrato. Estes cursos de água necessitam ser restaurados para poderem manter um equilíbrio ecológico aceitável, e continuarem fornecendo água em quantidade e qualidade adequadas (Devlin \& Brodie, 2005; Palmer \& Allan, 2006).

Em muitos países industrializados, onde há definição de prioridades para melhorar a qualidade e a condição ecológica das águas ocorre declínio no lançamento de poluentes nos cursos de água. Resultando uma redução das concentrações dos poluentes monitorados nestes cursos de água. A estabilização da concentração de poluentes e consequente melhora da qualidade das águas superficiais depende das prioridades definidas pelos gestores (Malmqvist \& Rundle, 2002).

Assumir a relevância da água como um bem natural, de interesse econômico e estratégico, é de extrema importância para todos os países, principalmente para o Brasil que possui aproximadamente $14 \%$ da disponibilidade mundial de água doce (Lanna, 2008). Para este autor, se a água for usada de maneira racional visando o desenvolvimento sustentável, o Brasil poderá obter vantagem competitiva.

Para Toledo e Nicolella (2002), as áreas agrícolas e regiões urbanas contribuem para o aumento na carga de fósforo nos recursos hídricos, e conforme Schwarzenbach et al. (2006), 60\% do fósforo presente nos recursos hídricos é de origem antropogênica. Este elemento em determinadas condições propicia 
a redução do oxigênio dissolvido, devido às reações de oxidação e consumo, o que limita a vida aquática.

O excesso de fósforo total pode vir a causar eutrofização em cursos d'água lênticos, visto que como um importante nutriente irá proporcionar o crescimento e desenvolvimento de plantas aquáticas, aumentando a competição entre essas plantas e os animais aquáticos pelo oxigênio dissolvido na água (Gonçalves et al., 2005; Moreira et al., 2010; Bittencourt \& Gobbi, 2006). Para Mansor (2005) o fósforo pode facilitar o crescimento de cianobactérias, que produzem toxinas que afetam a saúde humana.

Segundo Pignatti (2004) a presença excessiva de fósforo em cursos d'água, além de gerar eutrofização e crescimento de bactérias, pode proporcionar condições adequadas para a manutenção e procriação do vibrião do cólera, um causador de danos à saúde humana.

O excesso de poluição é prejudicial para quaisquer ecossistemas, e o excesso de manganês pode causar danos a saúde humana e animal, visto que, conforme Neves et al. (2009) é um nutriente importante para os seres vivos, porém, a sua assimilação excessiva via oral torna-o um elemento neurotóxico, podendo causar uma desordem crônica do Sistema Nervoso Central de maneira semelhante à doença de Parkinson.

Neves et al. (2009) identificaram que o manganês é bioacumulado nos seres humanos principalmente no fígado e pâncreas, devido às atividades metabólicas, e nos rins e intestinos devido às funções excretoras. De acordo com Zocche et al. (2010) e Veloso et al. (1995), o excesso de manganês tende a comprometer o desenvolvimento da flora e reduz a absorção de outros nutrientes importantes para o bom desempenho vegetal.

Existem vários modelos para avaliar as condições de transferência de poluentes abióticos presentes em um corpo hídrico para organismos aquáticos, para os processos ecológicos e condições ambientais (Monte, 2002), e modelos ecotoxicológicos, cobrindo a distribuição e os efeitos de algumas substâncias tóxicas (Jørgensen, 2005). Segundo este autor, há cerca de 100.000 produtos químicos utilizados no dia-a-dia dos países industrializados e aproximadamente 500 substâncias químicas tem uma avaliação dos riscos ambientais claramente analisados.

Em uma bacia que possui pontos de monitoramento da qualidade da água é de suma importância a modelagem da concentração dos contaminantes, visto que a biota, flutuante, fixa e com mobilidade existente, mantém contato com estes contaminantes em níveis heterogêneos de concentração ao longo do curso de água (Monte, 2002).
Park et al. (2008) efetuaram uma ampla revisão sobre alguns softwares de modelagem tais como AQUATOX, CASM, QUAL2K, WASP, GBMB e BASS, dentre outros, ou seja, sobre os principais modelos usados na análise dos efeitos da eutrofização, ecotoxicidade, dinâmica de materiais tóxicos, sedimentação e remineralização, bioacumulação e outras análises.

A elaboração de modelos que permitam prever o nível de contaminação existentes nos organismos em relação ao tempo pode ser conceitualmente complexa (Monte, 2002). Em bacias cujo volume de dados não permite utilizar modelos complexos, entende-se que se deva elaborar modelos capazes de, no mínimo, prever os níveis de concentração dos poluentes nos corpos hídricos.

A metodologia usada no modelo aqui proposto visou avaliar a inter-relação entre os poluentes existentes ao longo de um curso de água e os respectivos resultados de qualidade de água, de forma a identificar o comportamento de um dado poluente frente a possíveis alterações na sua concentração. Assim, os gestores podem propor ações para reduzir o impacto ambiental potencial e preservar as condições ambientais do entorno dos cursos de água.

Desta forma, este trabalho teve por objetivo desenvolver um modelo de dinâmica de sistemas para avaliar a variação da concentração de dois poluentes existentes na Bacia Hidrográfica do Rio das Mortes (BHRM), na Unidade de Planejamento e Gestão de Recursos Hídricos GD2 (UPGRH GD2). Os objetivos específicos são: i) desenvolver um modelo dinâmico para avaliar e prever a qualidade da água e o nível de poluição de bacias hidrográficas, ii) elaborar e validar o modelo dinâmico representativo para a concentração de poluentes existentes nos recursos hídricos na BHRM, iii) simular o comportamento da concentração dos poluentes Fósforo Total (PT) e Manganês Total (MnT) na BHRM e iv) efetuar modelagem de sensibilidade visando avaliar os efeitos do aumento ou redução de 20 e $50 \%$ nos valores das concentrações no primeiro ponto de monitoramento do Rio das Mortes e no ponto de monitoramento do Ribeirão Caieiro, mantidos inalterados os dados de vazão e concentração para todos os outros pontos de monitoramento.

\section{MATERIAIS E MÉTODOS}

A UPGRH GD2 é uma unidade de planejamento composta por três bacias hidrográficas dos rios do Cervo, Jacaré e das Mortes, localizada no Campo das Vertentes em Minas Gerais, entre as coordenadas geográficas $43^{\circ} 40^{\prime}$ e $45^{\circ} 20^{\prime} \mathrm{W}$ e $20^{\circ} 40^{\prime}$ e $21^{\circ} 40^{\prime} \mathrm{S}$ (Figura 1). Apenas a Bacia do Rio das Mortes possui um conjunto de estações de monitoramento de qualidade da 


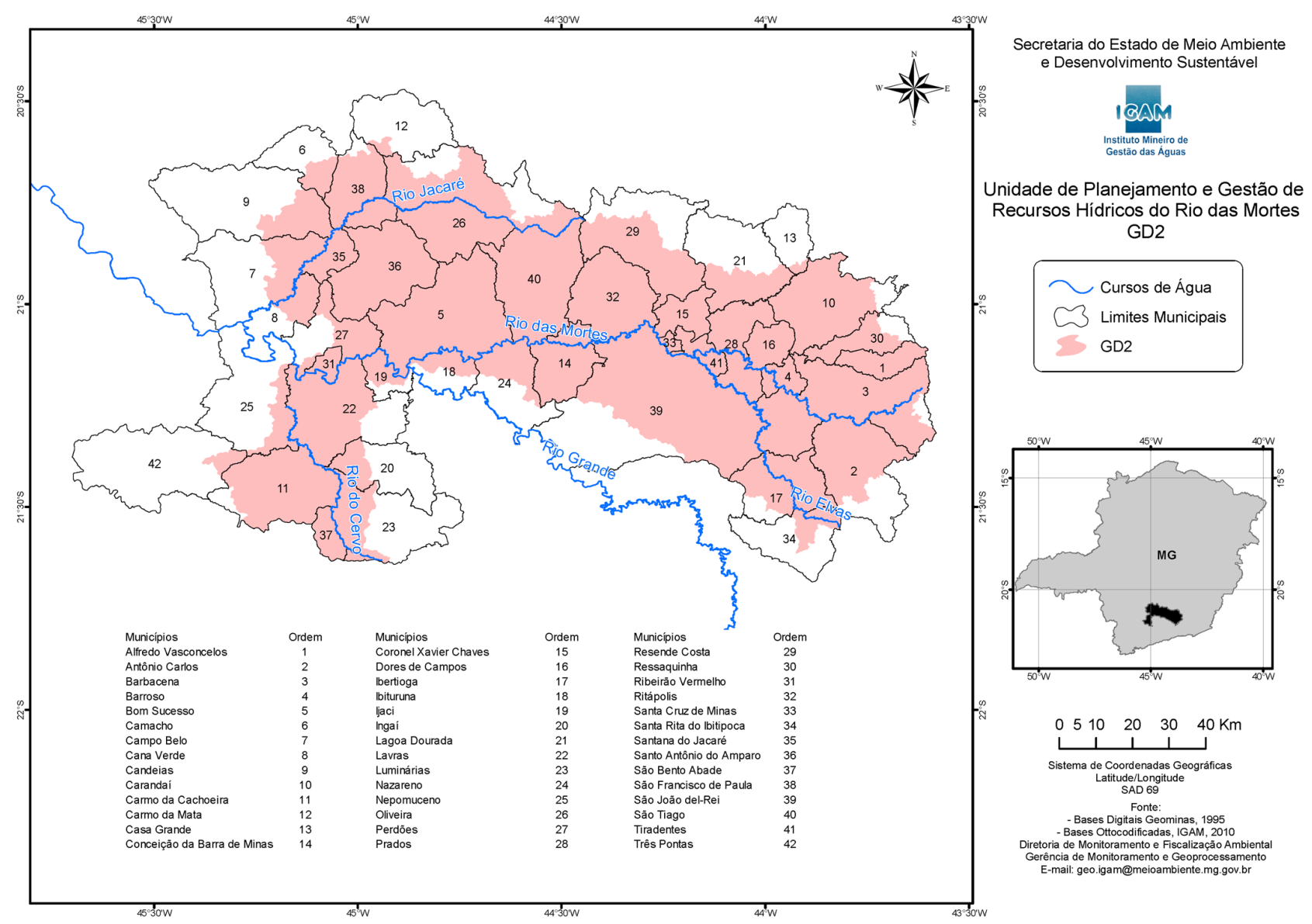

Figura 1 - Mapa da UPGRH GD2. Fonte: IGAM (2010).

água e medição de vazão com dados distribuídos em um espaço temporal maior.

Utilizaram-se os dados disponíveis na literatura sobre os valores espaciais e temporais do IQA e vazão na área da Bacia Hidrográfica do Rio das Mortes (BHRM) no estado de Minas Gerais. Os pontos de medição para os valores de vazão não coincidem com os pontos de monitoramento da qualidade da água na bacia no período de estudo. Assim, utilizaram-se os dados de:

- Vazões do Rio das Mortes medidas nas estações fluviométricas controladas pela ANA - HIDROWEB - (2010) e localizadas nas proximidades dos pontos de monitoramento de qualidade da água, entre os anos de:

- 1961 e 1965 para o BG011, na estação 61088000;

- 1998 e 2002 para BG012, BG013 e BG015, nas estações $610850000,61090000,6107000$ respectivamente;

- 1978 e 1982 para BG014, na estação 61089998;

- 1999 e 2003 para BG017, na estação 61135000;

- 2002 para BG010 fornecida pelo IGAM (2003a).

- Concentração de poluentes encontrada no relatório de qualidade das águas superficiais do Estado de Minas Gerais em 2000, fornecido pela FEAM e IGAM (2001).

- Concentração de poluentes encontrada nos relatórios de qualidade da água fornecidos pelo IGAM entre os anos de 2001 e 2009 (IGAM, 2002, 2003b, 2004, 2005, 2006, 2008 a, 2008 b, 2010).

O uso dos dados da vazão do Rio das Mortes em diferentes períodos deve-se ao fato da inexistência de um levantamento contínuo destas informações, não sendo possível coincidir os períodos de análise da qualidade da água com o monitoramento da vazão do Rio das Mortes. Assim, utilizaram-se os valores médios mensais das vazões existentes em um intervalo de tempo entre 1 e 5 anos de monitoramento contínuo.

Cumpre citar que nos estudos realizados pela Fundação Rural Mineira (2005) e pela Fundação Centro Tecnológico de Minas Gerais - CETEC (1988), sobre a BHRM, esta bacia caracteriza-se como uma região homogênea para a precipitação média anual, com variação entre 1285 e 1420 mm/ano. O uso dos valores de precipitação e evaporação na bacia foram simplificados visto que as medições das concentrações dos poluentes estão distribuídas em períodos chuvosos e secos. 
Os cálculos tiveram por base os resultados obtidos nas campanhas de monitoramento de qualidade da água nos anos entre 2000 e 2009, fornecidos pelo IGAM - Instituto Mineiro de Gestão das Águas, e FEAM - Fundação Estadual do Meio Ambiente, originalmente medidos em miligramas por litro (mg/L). Estes poluentes possuem valores máximos para rios enquadrados na Classe 2 conforme DNC COPAM/ CERH-MG no 1, de 05 de Maio de 2008, de 0,100mg/L $\left(100 \mathrm{mg} / \mathrm{m}^{3}\right)$ (FEAM, IGAM, 2001; IGAM, 2002, 2003b, 2004, 2005, 2006, 2008a, 2008 b, 2010; MINAS GERAIS, 2008).

O estudo foi realizado com os dados da BHRM, que abrange uma área de $6606 \mathrm{~km}^{2}$, localizada entre as coordenadas geográficas $43^{\circ} 40^{\prime}$ e $44^{\circ} 50^{\prime} \mathrm{W}$, e $21^{\circ} 00^{\prime}$ e $21^{\circ} 30^{\prime} S$, e possui estudos de caracterização ambiental desde 1988 (CETEC, 1988; IGAM, 2010).

A UPGRH GD2 apresenta os pontos de monitoramento BG010 até BG017 pertencentes à BHRM (Figura 2). O ponto BG019 está localizado no Rio Grande, sendo o quarto ponto de monitoramento desse rio. O BG021 é o único ponto de monitoramento do Rio Jacaré, e o Rio do Cervo não possui pontos de monitoramento de qualidade da água.

Para desenvolver uma modelagem dinâmica que avalie o comportamento de poluentes existentes na BHRM, foram utilizados os dados dos pontos de monitoramento BG010, BG011, BG012, BG013, BG014, BG015 e BG017 distribuídos na área de estudo conforme a Figura 2.

\section{Modelagem Dinâmica}

Para efetuar uma análise ambiental de um rio é necessário calcular a vazão mássica (carga) e a taxa de aporte ou degradação dos poluentes entre os pontos de monitoramento. No estado de Minas Gerais os resultados obtidos devem enquadrar-se legalmente conforme a DNC COPAM/CERH-MG $n^{\circ} 1$, de 05 de Maio de 2008 (MINAS GERAIS, 2008).

Para o desenvolvimento de um modelo dinâmico para avaliar e prever a qualidade da água e o nível

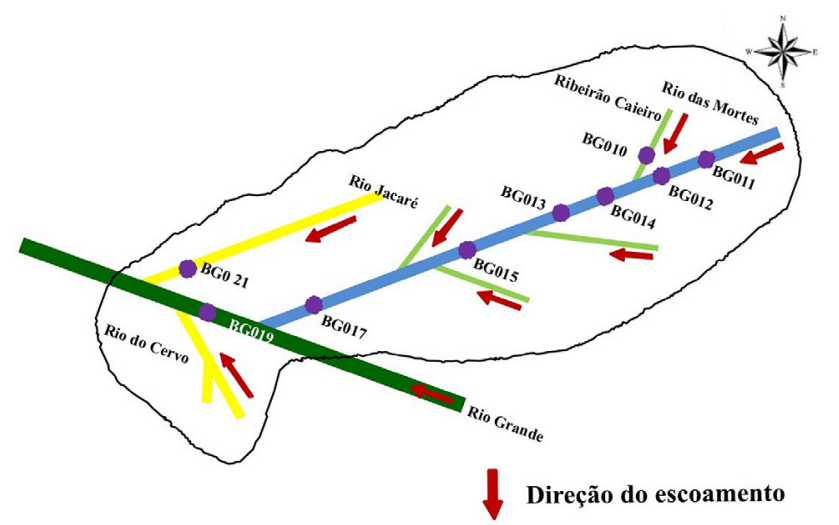

Figura 2 - Modelo esquemático dos Pontos de monitoramento na UPGRH GD2. de poluição de bacias hidrográficas utilizou-se o software Stella da empresa ISee Systems. Este software foi escolhido por ser mundialmente utilizado para simulações de sistemas associados a sustentabilidade, cuja origem são os trabalhos pioneiros em dinâmica de sistemas do consagrado Professor Jay Forrester, do Massachusetts Institute of Technology, MIT.

Assim, o software STELLA foi utilizado para desenvolver o modelo em que os elementos de vazão e concentração de poluentes interconectam-se para explicar a dinâmica do comportamento da carga poluente ao longo das estações de monitoramento. A Figura 3 representa o diagrama de modelagem da concentração de poluentes em um rio.

Considerou-se que as concentrações dos poluentes escolhidos são homogêneas nos pontos de monitoramento devido aos movimentos turbulentos existentes em meio lótico. Desta forma os valores de concentração representam todo o material adicionado ao curso de água por fontes pontuais e difusas a montante dos pontos de monitoramento.

Em um ponto de monitoramento a vazão mássica (carga) (mipol) de um poluente, em mg/dia, é dada por:

$$
\dot{\mathrm{m}} \_ \text {pol }=\mathrm{c} \_\mathrm{p} \times \mathrm{Q}
$$

em que $\mathrm{cp}=$ concentração de poluente no ponto de monitoramento $\left(\mathrm{mg} / \mathrm{m}^{3}\right)$ e $\mathrm{Q}=$ vazão volumétrica no ponto de monitoramento ( $\left.\mathrm{m}^{3} / \mathrm{dia}\right)$.

A BHRM não possui estudos sobre a variação da concentração de poluentes entre os pontos de monitoramento da qualidade da água, BG010, BG011, BG012, BG013, BG014, BG015 e BG017. A variação de aporte ou decaimento da concentração de poluente, $\operatorname{Tax}_{A D}$, será determinada pela diferença da concentração entre os pontos de monitoramento $\mathrm{XeY}$ multiplicada pela vazão do ponto de monitoramento $X$.

$$
\operatorname{Tax}_{A D}=\Delta C_{p} \times Q_{x}
$$

em que, $\Delta c_{p}=c_{x}-c_{y} ; c_{x}=$ concentração do poluente no ponto $X$ de monitoramento, $c_{y}=$ concentração no ponto $\mathrm{Y}$ a jusante do ponto $\mathrm{X}$ de monitoramento, $Q_{x}=$ vazão do rio no ponto próximo ao ponto $X$ de monitoramento.

A equação de equilíbrio que representa a concentração de poluente em qualquer ponto de monitoramento (c_p) é dada por

$$
C_{p} Q=C_{n} Q_{n}+T_{a x}
$$

em que, $c_{p}$ e $c_{n}$ são, respectivamente, a concentração de poluente final e no ponto de monitoramento $n ; \mathrm{Q}$ e $\mathrm{Q}_{\mathrm{n}}$ são, respectivamente, a vazão volumétrica final e vazão no ponto de monitoramento $n$. 


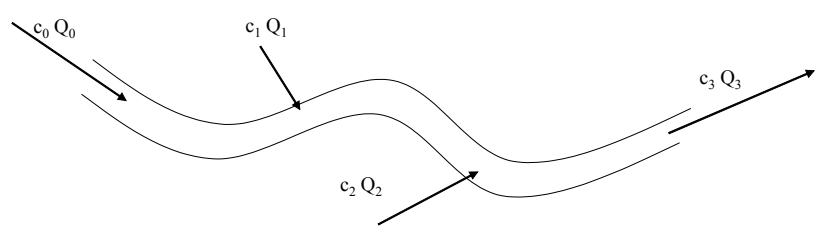

Figura 3 - Diagrama de modelagem para a concentração de poluentes em um rio.

Considerando que $c_{p} Q=\dot{m}_{p}, c_{n} Q_{n}=\dot{m}_{p n}$ e $\operatorname{Tax}_{A D}$ $=\dot{m}_{\mathrm{pc}}$ a equação (3) pode ser reescrita em termos de vazão mássica como:

$$
\dot{m}_{\mathrm{p}}=\dot{\mathrm{m}}_{\mathrm{pn}}+\dot{\mathrm{m}}_{\mathrm{pc}}
$$

Estas equações foram colocadas nos conversores e fluxos do software STELLA.

O modelo foi validado tendo por base dados reais da BHRM de vazão volumétrica e concentração de poluentes existentes nos recursos hídricos. As simulações serviram, em termos de validação, para analisar o comportamento da concentração dos poluentes Fósforo Total (PT) e Manganês Total (MnT) na BHRM, no período entre 1999 e 2008.

\section{RESULTADOS E DISCUSSÃO}

Foram realizadas três modelagens de sensibilidade para as alterações na concentração dos poluentes Manganês Total e Fósforo Total:

- Modelagem A - alteração dos valores da concentração apenas do ponto de monitoramento BG011.

- Modelagem B - alteração dos valores da concentração apenas do ponto de monitoramento BG010.

- Modelagem C - alteração dos valores da concentração nos pontos de monitoramento BG010 e 11.

Em cada modelagem foram realizadas quatro subdivisões:

- A0, BO e CO - sem alteração do valor de concentração;

- A1, B1 e C1 - redução de $20 \%$ no valor de concentração;

- A2, B2 e C2 - redução de $50 \%$ no valor de concentração;

- A3, B3 e C3 - aumento de $20 \%$ no valor de concentração;

- A4, B4 e C4 - aumento de 50\% no valor de concentração.

Cumpre citar que nas modelagens de redução do valor de concentração dos poluentes, não se considerou o limite natural (Background) para estes compostos, visto que não foi encontrada nenhuma pesquisa ou bibliografia que trate especificamente dos limites naturais dos compostos de Fósforo e Manganês nos cursos de água da BHRM. Mas, conforme Menezes et al. (2009) e Marchesan et al. (2009) os valores naturais para manganês e fósforo não ultrapassam 0,2 $\mathrm{mg} / \mathrm{L}\left(200 \mathrm{mg} / \mathrm{m}^{3}\right)$ na maioria das águas superficiais brasileiras, entretanto a legislação brasileira trabalha com $0,1 \mathrm{mg} / \mathrm{L}\left(100 \mathrm{mg} / \mathrm{m}^{3}\right)$ como limite máximo.

\section{Análise dos resultados para o poluente Manganês Total (MnT)}

Conforme o IGAM (2010) a concentração do poluente manganês está associada à "ausência de cobertura vegetal na região, o uso e o manejo inadequado do solo, além do revolvimento do sedimento dos corpos de água através da extração de areia", e da existência de processos erosivos nas margens dos cursos de água.

Os resultados da modelagem de sensibilidade $A$ na BHRM para a concentração do poluente MnT, nos pontos de monitoramento BG011, BG014 e BG017, são apresentados na Tabela 1. Observa-se que apesar da grande variação no ponto BG011, entre -25,2 e $72,2 \%$, os pontos BG014 e BG017 apresentaram uma variação em relação a modelagem $A 0$, entre $-0,6$ e $3,6 \%$ e 0,0 e $0,4 \%$ respectivamente.

Ao analisar a modelagem de sensibilidade B na BHRM para a concentração do poluente MnT, nos pontos de monitoramento BG010, BG014 e BG017, conforme a Tabela 2, observa-se que apesar de o ponto BG010 responder coerentemente às alterações impostas, entre $-23,7$ e $59,4 \%$, os pontos BG014 e BG017 variaram em relação a modelagem B0 entre $-4,2$ e $11,4 \%$ e $-0,3$ e $1,2 \%$, respectivamente.

Ao analisar a modelagem de sensibilidade $C$ na BHRM para a concentração do poluente $\mathrm{MnT}$, nos pontos de monitoramento BG011, BG010, BG014 e BG017, apresentado na Tabela 3, observa-se que apesar de os pontos BG010 e BG011 apresentarem alterações coerentes com o percentual de alteração, os pontos BG014 e BG017 variaram em relação a modelagem C0 entre $-4,8$ e $15,1 \%$ e $-0,4$ e $1,7 \%$, respectivamente.

A grande distância entre os pontos de monitoramento analisados (Tabela 4) é um fator que influencia nos resultados da variação de concentração entre os pontos BG010 e BG014, BG010 e BG017, BG011 e BG014, BG011 e BG017 nas três modelagens. Este resultado de simulação indica que existem outros mecanismos dinâmicos ao longo do curso d'água que agem sobre a concentração do poluente Manganês Total presente na BHRM.

\section{Análise dos resultados para o poluente Fósforo Total}

Conforme o IGAM (2010), a presença de fósforo está relacionada ao uso de fertilizantes nas atividades agrícolas existentes na BHRM, sendo originário do 
Tabela 1 - Resultados médios para a modelagem de sensibilidade A, para o poluente $\mathrm{MnT}$, nos pontos de monitoramento na BHRM $\left(\mathrm{mg} / \mathrm{m}^{3}\right)$.

\begin{tabular}{cccc}
\hline \hline & BG011 & BG014 & BG017 \\
\hline A0 & 89,620 & 151,650 & 271,152 \\
A1 & 67,061 & 150,712 & 271,084 \\
A2 & 38,563 & 150,563 & 271,068 \\
A3 & 114,285 & 153,443 & 271,388 \\
A4 & 154,302 & 157,100 & 272,156 \\
\hline \hline
\end{tabular}

Tabela 2 - Resultados médios para a modelagem de sensibilidade $\mathrm{B}$, para o poluente $\mathrm{MnT}$, nos pontos de monitoramento na BHRM $\left(\mathrm{mg} / \mathrm{m}^{3}\right)$.

\begin{tabular}{cccc}
\hline \hline & BG010 & BG014 & BG017 \\
\hline B0 & 548,797 & 151,650 & 271,152 \\
B1 & 418,898 & 145,231 & 270,241 \\
B2 & 226,474 & 136,312 & 269,285 \\
B3 & 678,970 & 158,465 & 272,291 \\
B4 & 874,700 & 168,954 & 274,333 \\
\hline \hline
\end{tabular}

Tabela 3 - Resultados médios para a modelagem de sensibilidade $\mathrm{C}$, para o poluente $\mathrm{MnT}$, nos pontos de monitoramento na BHRM $\left(\mathrm{mg} / \mathrm{m}^{3}\right)$.

\begin{tabular}{ccccc}
\hline \hline & BG011 & BG010 & BG014 & BG017 \\
\hline C0 & 89,620 & 548,797 & 151,789 & 271,398 \\
C1 & 67,061 & 418,898 & 144,494 & 270,434 \\
C2 & 38,563 & 226,474 & 135,682 & 269,494 \\
C3 & 114,285 & 678,970 & 160,435 & 272,882 \\
C4 & 154,302 & 874,700 & 174,771 & 276,022 \\
\hline \hline
\end{tabular}

escoamento superficial e sub-superficial, e de fontes difusas. Esta bacia apresentou índices elevados deste poluente no ano de 2009, no período de estiagem, indicando a existência de fontes pontuais significantes.

Os resultados da modelagem de sensibilidade $A$ na BHRM para a concentração do poluente fósforo total (PT), nos pontos de monitoramento BG011, BG014 e BG017 (Tabela 5) mostram que apesar da variação no ponto BG011, de $-24,2$ e $69,9 \%$, os pontos BG014 e BG017 apresentam variação em relação a modelagem $A 0$, entre 0,0 e $0,5 \%$ e 0,0 e $0,1 \%$ respectivamente.

Ao analisar da modelagem de sensibilidade $B$ na BHRM para a concentração do poluente PT, nos pontos de monitoramento BG010, BG014 e BG017 (Tabela 6) nota-se que o ponto BG010 variou de maneira coerente com as alterações impostas, -22,9 e $58,2 \%$; no entanto os pontos BG014 e BG017 variaram em relação a modelagem B0 entre -2,2 e 7,5\% e -0,7 e 2,3\% respectivamente.

Ao analisar a modelagem de sensibilidade $C$ na BHRM para a concentração do poluente $\mathrm{PT}$, nos pontos de monitoramento BG011, BG010, BG014 e BG017 (Tabela 7), observa-se que os pontos BG010 e BG011 apresentaram alterações coerentes com o percentual de alteração proposto, mas os pontos BG014 e BG017 variaram em relação a modelagem C0, entre $-2,2$ e $8,6 \%$ e $-0,7$ e $2,6 \%$ respectivamente, de maneira muito semelhante ao teste $B$.

Da mesma maneira que a distância entre os pontos de monitoramento analisados influenciou na simulação para o poluente manganês total, esta também influenciou os resultados da simulação para fósforo.

A distância é um fator que influencia os resultados entre os pontos analisados BG010 e BG014, BG010 e BG017, BG011 e BG014, BG011 e BG017 nas três modelagens para o poluente fósforo total, indicando a existência de outros mecanismos dinâmicos ao longo do curso d'água que agem sobre a concentração do poluente PT presente na BHRM.

A diferença da vazão nos pontos de monitoramento BG010, máxima de $5,67 \mathrm{~m}^{3} / \mathrm{s}$, BG011, máxima de $68 \mathrm{~m}^{3} / \mathrm{s}$, e no BG014, máxima de $86,47 \mathrm{~m}^{3} / \mathrm{s}$, pode ser um dos fatores que influenciaram a variação na concentração dos poluentes manganês total e fósforo total, entre os pontos BG010 e BG014, e BG011 e BG014. Ressalte-se que o processo de diluição ocorre em uma maior taxa quando a vazão é maior. Esta situação provavelmente ocorre devido ao aporte hídrico de outros afluentes não identificados e monitorados, que podem contribuir com uma menor ou maior concentração destes poluentes.

Apesar da variação de concentração nas modelagens $A, B$ e $C$ nos pontos de monitoramento BG010 e BG011, esta é compensada pela maior vazão existente nos pontos de monitoramento BG014 e BG017, que promovem a diluição ao longo do curso d'água do Rio das Mortes.

Os resultados das modelagens de sensibilidade A1 até A4, B1 até B4, e C1 até C4 para os pontos de monitoramento analisados, variaram aproximadamente entre 150 e $270 \mathrm{mg} / \mathrm{m}^{3}$ para manganês total, e entre 111 e $140 \mathrm{mg} / \mathrm{m}^{3}$ para fósforo total. Estes resul-

Tabela 4 - Distância entre os pontos de monitoramento analisados na BHRM, mensuradas através do Google Earth (2010).

\begin{tabular}{ccc}
\hline \hline De & Para & Distância $(m)^{*}$ \\
\hline BG011 & BG014 & 69.200 \\
BG011 & BG017 & 238.100 \\
BG010 & BG014 & 14.700 \\
BG010 & BG017 & 183.600 \\
\hline \hline valor aproximado & &
\end{tabular}

* valor aproximado 
Tabela 5 - Resultados médios para a modelagem de sensibilidade A, para o poluente PT, nos pontos de monitoramento na BHRM $\left(\mathrm{mg} / \mathrm{m}^{3}\right)$.

\begin{tabular}{lccc}
\hline \hline & BG011 & BG014 & BG017 \\
\hline A0 & 61,519 & 116,749 & 136,542 \\
A1 & 46,651 & 116,724 & 136,542 \\
A2 & 26,695 & 116,724 & 136,542 \\
A3 & 77,977 & 116,844 & 136,558 \\
A4 & 104,496 & 117,310 & 136,661 \\
\hline \hline
\end{tabular}

Tabela 6 - Resultados médios para a modelagem de sensibilidade $\mathrm{B}$, para o poluente $\mathrm{PT}$, nos pontos de monitoramento na BHRM $\left(\mathrm{mg} / \mathrm{m}^{3}\right)$.

\begin{tabular}{cccc}
\hline \hline & BG010 & BG014 & BG017 \\
\hline B0 & 440,670 & 116,749 & 136,542 \\
B1 & 339,963 & 114,187 & 135,533 \\
B2 & 191,416 & 111,460 & 134,270 \\
B3 & 542,746 & 119,850 & 137,689 \\
B4 & 697,310 & 125,518 & 139,689 \\
\hline \hline
\end{tabular}

Tabela 7 - Resultados médios para a modelagem de sensibilidade $\mathrm{C}$, para o poluente $\mathrm{PT}$, nos pontos de monitoramento na BHRM $\left(\mathrm{mg} / \mathrm{m}^{3}\right)$.

\begin{tabular}{ccccc}
\hline \hline & BG011 & BG010 & BG014 & BG017 \\
\hline C0 & 61,519 & 440,670 & 116,749 & 136,542 \\
C1 & 46,651 & 339,963 & 114,179 & 135,533 \\
C2 & 26,695 & 191,416 & 111,452 & 134,270 \\
C3 & 77,977 & 542,746 & 120,037 & 137,727 \\
C4 & 104,496 & 697,310 & 126,731 & 140,082 \\
\hline \hline
\end{tabular}

tados estão acima do limite máximo permitido, para manganês total e fósforo total, de acordo com a DNC COPAM/CERH-MG n 1 , de 05 de Maio de 2008, para cursos d'água enquadrados como classe 2 , cujo limite é de $0,1 \mathrm{mg} / \mathrm{L}\left(100 \mathrm{mg} / \mathrm{m}^{3}\right)$ (MINAS GERAIS, 2008), indicando a necessidade de um enquadramento diferente do atual.

\section{CONCLUSÕES}

Em observância aos objetivos propostos e dados obtidos e analisados, este trabalho apresenta, como principais realizações e conclusões.

Como uma aplicação prática e importante do processo de estabelecimento e validação do modelo, efetuaram-se testes de sensibilidade visando avaliar os efeitos do aumento ou redução de 20 e $50 \%$ nos valores das concentrações no primeiro ponto de monitoramento do Rio das Mortes e no ponto de monitoramento do Ribeirão Caieiro, mantidos inalterados os dados de vazão e concentração para todos os outros pontos de monitoramento.

Os testes de sensibilidade referentes ao teor de Manganês Total e Fósforo Total mostraram que as respostas às variações pontuais, positivas ou negativas, de MnT e PT são não lineares e, para os valores acrescidos ou aumentados, há pouca variação em pontos de observação a jusante. Este resultado de simulação indica que existem outros mecanismos dinâmicos ao longo do curso d'água que agem sobre a concentração do poluente Manganês total presente na BHRM, dentre os quais o grande volume de água, a absorção pela margem e fundos da bacia e pela quantidade relativamente pequena de variação de concentração. Os dados mostraram claramente a sensibilidade do modelo a pequenas taxas de variações que, apesar de baixas, podem levar a valores inaceitáveis de poluição.

Os resultados da modelagem para os poluentes manganês total e fósforo total, no trecho compreendido entre os pontos de monitoramento analisados, apresentam valores acima do permitido para o atual enquadramento, classe 2 , comprometendo a qualidade das águas na simulação, desta maneira o modelo poderá servir como ferramenta de gestão para prever possível alterações dos poluentes analisados.

\section{REFERÊNCIAS}

ANA - Agência Nacional de Águas. HIDROWEB. 2010. Disponível em <http://www.hidroweb.ana.gov.br> Acessado em 10 abr. 2010.

Bittencourt, S. \& Gobbi, E.F. 2006. Carga máxima de fósforo admissível ao reservatório Piraquara II, uma aplicação do processo TMDL. Rev. Bras. Ciênc. Solo 30(3): 595-603.

CETEC - Fundação Centro Tecnológico de Minas Gerais. 1988. Caracterização ambiental da bacia do Rio das Mortes: relatório técnico final: etapa 1. Belo Horizonte: Fundação Centro Tecnológico de Minas Gerais, p. 148-377.

Devlin, M.J. \& Brodie, J. 2005. Terrestrial discharge into the Great Barrier Reef Lagoon: nutrient behavior in coastal waters. Marine Pollution Bulletin 51: 9-22.

FEAM - Fundação Estadual do Meio Ambiente \& IGAM - Instituto Mineiro de Gestão das Águas. 2001. Relatório de qualidade das águas superficiais do Estado de Minas Gerais em 2000: projeto sistema de monitoramento da qualidade das águas superficiais do Estado de Minas Gerais. Belo 
Horizonte: Fundação Estadual do Meio Ambiente, Instituto Mineiro de Gestão das Águas, 346p.

Fundação Rural Mineira. 2005. Atlas digital das águas de Minas: uma ferramenta para o planejamento e gestão dos recursos hídricos. Coordenação técnica, direção e roteirização Humberto Paulo Euclydes. Belo Horizonte: RURALMINAS; Viçosa: UFV. 1 CD-ROM + 1 manual (78p.). ISBN 85-7601-082-8.

Gonçalves, C.S.; Rheinheimer, D.S.; Pellegrini, J.B.R. \& Kist, S.L. 2005. Qualidade da água numa microbacia hidrográfica de cabeceira situada em região produtora de fumo. Rev. Bras. Eng. Agríc. Ambient. 9(3): 391-399.

Google Earth: programa da Internet. Versão 6.0. Google, 2010. Disponível em <earth.google.com. br/>. Acessado em: 21 set. 2010.

IGAM - Instituto Mineiro de Gestão das Águas. 2002. Qualidade das águas superficiais no Estado de Minas em 2001. Belo Horizonte: Instituto Mineiro de Gestão das Águas. 205p.

IGAM - Instituto Mineiro de Gestão das Águas. 2003a. Águas de Minas. Belo Horizonte: Instituto Mineiro de Gestão das Águas. CD-ROM.

IGAM - Instituto Mineiro de Gestão das Águas. 2003b. Relatório de monitoramento das águas superficiais na Bacia do Rio Grande em 2002. Belo Horizonte: Instituto Mineiro de Gestão das Águas. 245p.

IGAM - Instituto Mineiro de Gestão das Águas. 2004. Relatório de monitoramento das águas superficiais na Bacia do Rio Grande em 2003. Belo Horizonte: Instituto Mineiro de Gestão das Águas. 262p.

IGAM - Instituto Mineiro de Gestão das Águas. 2005. Relatório de monitoramento das águas superficiais na Bacia do Rio Grande em 2004. Belo Horizonte: Instituto Mineiro de Gestão das Águas. 248p.

IGAM - Instituto Mineiro de Gestão das Águas. 2006. Relatório de monitoramento das águas superficiais na Bacia do Rio Grande em 2005. Belo Horizonte: Instituto Mineiro de Gestão das Águas. 164p.

IGAM - Instituto Mineiro de Gestão das Águas. 2007. Monitoramento da qualidade das águas superficiais na Bacia do Rio Grande em 2006. Belo Horizonte: Instituto Mineiro de Gestão das Águas. 183p.

IGAM - Instituto Mineiro de Gestão das Águas. 2008a. Monitoramento da qualidade das águas superficiais na Bacia do Rio Grande em 2007. Belo Horizonte: Instituto Mineiro de Gestão das Águas. 196p.

IGAM - Instituto Mineiro de Gestão das Águas. 2008b. Monitoramento da qualidade das águas superficiais na Bacia do Rio Grande em 2008. Belo Horizonte: Instituto Mineiro de Gestão das Águas. 304p.
IGAM - Instituto Mineiro de Gestão das Águas. 2010. Monitoramento da qualidade das águas superficiais na Bacia do Rio Grande em 2009. Belo Horizonte: Instituto Mineiro de Gestão das Águas. 312p.

Jørgensen, S.E. 2005. Ecological Modelling: editorial overview 2000-2005. Ecological Modelling 188(2-4):137-144.

Lanna, A.E.L. 2008. A economia dos recursos hídricos: os desafios da alocação eficiente de um recurso (cada vez mais) escasso. Estud. Av. 22(63): 113-130.

Malmqvist, B. \& Rundle, S. 2002. Threats to the running water ecosystems of the world. Environmental Conservation 29(2): 134-153.

Mansor, M.T.C. 2005. Potencial de poluição de águas superficiais por fontes não pontuais de fósforo na Bacia Hidrográfica do Ribeirão do Pinhal, Limeira, SP. Tese de doutorado. Universidade Estadual de Campinas - UNICAMP. $189 \mathrm{f}$.

Marchesan, E.; Sartori, G. M. S.; Reimche, G. B.; Avila, L. A.; Zanella, R.; Machado, S. L. de O.; Macedo, V. R. M. \& Cogo, J. P. 2009. Qualidade de água dos rios Vacacaí e Vacacaí-Mirim no Estado do Rio Grande do Sul, Brasil. Cienc. Rural [online] 39(7): 2050-2056.

Menezes, J. M.; Prado, R. B.; Silva Júnior, G. C. da; Mansur, K. L. \& Oliveira, E. S. 2009. Qualidade da água e sua relação espacial com as fontes de contaminação antrópicas e naturais: bacia hidrográfica do rio São Domingos - RJ. Eng. Agríc. [online] 29(4): 687-698.

MINAS GERAIS. Secretaria de Estado de Meio Ambiente e Desenvolvimento Sustentável. 2008. Deliberação Normativa Conjunta COPAM/CERHMG $n^{\circ} 1$, de 05 de Maio de 2008. Legislação Estadual de Recursos Hídricos. Disponível em http://www.semad.mg.gov.br. Acessado em 15 mai. 2009.

Monte, L. 2002. A methodology for modeling the contamination of moving organisms in water bodies with spatial and time dependent pollution levels. Ecol. Model. 58(1-2): 21-33.

Moreira, J.A.; Vitti, D.M.S.S.; Patino, R.M.; Silva, T.S.; Bueno, I.C. \& Berenchtein, B. 2010. Impacto ambiental provocado pelo $\mathrm{P}$ em dietas suplementadas com enzima fitase e proteína ideal para suínos em crescimento: estudo do fluxo do $\mathrm{P}$ no metabolismo animal. Arq. Bras. Med. Vet. Zootec. 62(5): 1206-1215.

Neves, E.B.; Mendonca Junior, N. \& Moreira, M.F.R. 2009. Avaliação da exposição a metais numa oficina de recuperação de armamento de uma organização militar. Ciênc. Saúde Coletiva 14(6): 2269-2280. 
Palmer, M.A. \& Allan, J.D. 2006. Restoring Rivers. National Academy of Science: Issues Sci.Technol. (22): 40-48.

Park, R.A.; Clough, J.S. \& Wellman, M.C. 2008. AQUATOX: Modeling environmental fate and ecological effects in aquatic ecosystems. Ecol. Model. 213(1): 1-15.

Pignatti, M.G. 2004. Saúde e ambiente: as doenças emergentes no Brasil. Ambient. Soc. 7(1): 133-147.

Schwarzenbach, R.P.; Escher, B.I.; Fenner, K.; Hofstetter, T.B.; Johnson, C.A.; von Gunten, U. \& Wehrli, B. 2006. The Challenge of Micropollutants in Aquatic Systems. Science 313(5790): 1072-1077.

Toledo, L.G. \& Nicolella, G. 2002. Índice de Qualidade de água em microbacia sob uso agrícola e urbano. Sci. Agric. 59(1): 181-186.
Veloso, C.A.C.; Muraoka.T.; Malavolta, E. \& Carvalho, J.G. 1995. Influência do manganês sobre a nutrição mineral e crescimento da pimenteira do reino (Piper nigrum, L.). Sci. Agric. 52(2): 376-383.

Zocche, J.J.; Freitas, M. \& Quadros, K.E. 2010. Concentração de $\mathrm{Zn}$ e $\mathrm{Mn}$ nos efluentes do beneficiamento de carvão mineral e em Typha domingensis Pers (TYPHACEAE). Rev. Árvore [online] 34(1): 177-188. 